2015

\title{
From chemical systems to systems chemistry: Patterns in space and time
}

Kenneth Showalter

Irving R. Epstein

Follow this and additional works at: https://researchrepository.wvu.edu/faculty_publications

\section{Digital Commons Citation}

Showalter, Kenneth and Epstein, Irving R., "From chemical systems to systems chemistry: Patterns in space and time" (2015). Faculty Scholarship. 175.

https://researchrepository.wvu.edu/faculty_publications/175

This Article is brought to you for free and open access by The Research Repository @ WVU. It has been accepted for inclusion in Faculty Scholarship by an authorized administrator of The Research Repository @ WVU. For more information, please contact ian.harmon@mail.wvu.edu. 


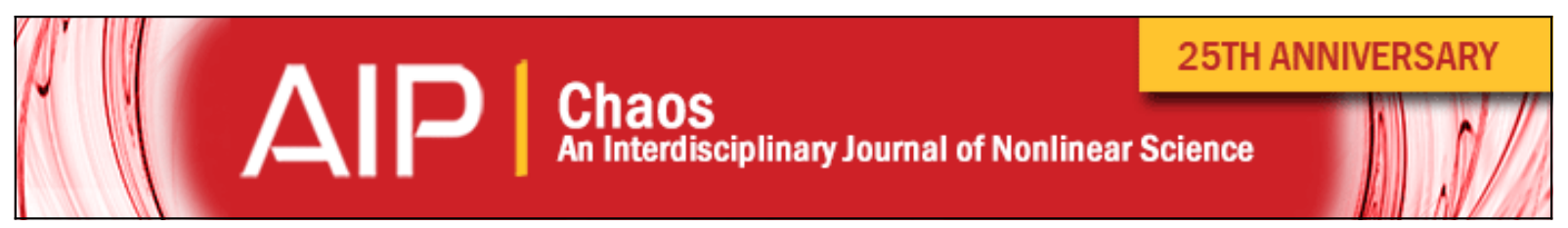

\section{From chemical systems to systems chemistry: Patterns in space and time}

Kenneth Showalter and Irving R. Epstein

Citation: Chaos: An Interdisciplinary Journal of Nonlinear Science 25, 097613 (2015); doi: 10.1063/1.4918601

View online: http://dx.doi.org/10.1063/1.4918601

View Table of Contents: http://scitation.aip.org/content/aip/journal/chaos/25/9?ver=pdfcov

Published by the AIP Publishing

\section{Articles you may be interested in}

Complex network from time series based on phase space reconstruction

Chaos 19, 033137 (2009); 10.1063/1.3227736

Transition from phase to generalized synchronization in time-delay systems

Chaos 18, 023118 (2008); 10.1063/1.2911541

Characterization of patterns, oscillations, and chaos in chemical systems

J. Chem. Phys. 115, 8327 (2001); 10.1063/1.1410378

On the deduction of chemical reaction pathways from measurements of time series of concentrations

Chaos 11, 108 (2001); 10.1063/1.1336499

Nonlinear prediction, filtering, and control of chemical systems from time series

Chaos 7, 614 (1997); 10.1063/1.166261

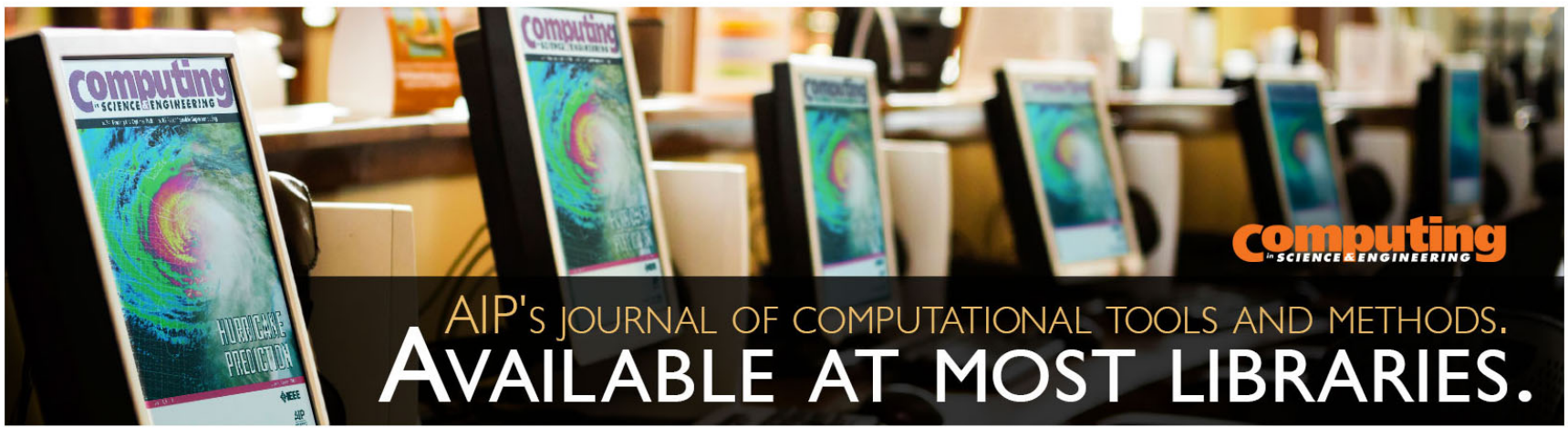




\title{
From chemical systems to systems chemistry: Patterns in space and time
}

\author{
Kenneth Showalter ${ }^{1}$ and Irving R. Epstein ${ }^{2}$ \\ ${ }^{1}$ C. Eugene Bennett Department of Chemistry, West Virginia University, Morgantown, \\ West Virginia 26506-6045, USA \\ ${ }^{2}$ Department of Chemistry and Volen National Center for Complex Systems, Brandeis University, Waltham, \\ Massachusetts 02454-9110, USA
}

(Received 30 December 2014; accepted 7 April 2015; published online 29 April 2015)

\begin{abstract}
We present a brief, idiosyncratic overview of the past quarter century of progress in nonlinear chemical dynamics and discuss what we view as the most exciting recent developments and some challenges and likely areas of progress in the next 25 years. (C) 2015 AIP Publishing LLC.

[http://dx.doi.org/10.1063/1.4918601]
\end{abstract}

Chemical systems display a remarkable range of nonlinear phenomena in time and space. These include temporal oscillations, multistability and chaos as well as stationary (Turing) spatial patterns, and a variety of traveling and standing waves. Chaos was founded soon after a series of developments that turned nonlinear chemical dynamics from a field whose subject matter consisted largely of a set of unconnected, often serendipitous observations to one in which one could hope to understand and even design phenomena whose existence was previously viewed with skepticism by much of the scientific community. During the past twenty-five years, experimental and theoretical chemists have learned much about the properties and mechanisms associated with these strange and beautiful patterns, and they have begun to link their understanding with discoveries in biology, physics, engineering, and other fields. There remain many challenges for those who seek to weave together chemical reactions, transport phenomena, and external forces in new and exciting ways.

\section{INTRODUCTION-EARLY DEVELOPMENTS}

Independent and virtually simultaneous events in England and the Soviet Union inadvertently initiated what has become the research "field" of nonlinear chemical dynamics. In 1951, Boris P. Belousov submitted a paper on experimental observations of oscillations in a chemical reaction, and Alan Turing submitted a theoretical paper on the chemical basis of morphogenesis. Belousov's paper was rejected, with the editor commenting "that his 'supposedly discovered discovery' was quite impossible," 1 and, after a second rejection, was finally published in a booklet on radiology by his own institution.,3 Anatol M. Zhabotinsky refined and improved on Belousov's discovery, ${ }^{1,4,5}$ bringing this strange and seemingly "alive" chemical reaction to the forefront of modern studies on complexity and nonlinear dynamics. The remarkable properties of the BelousovZhabotinsky (BZ) reaction have made it perhaps one of the most studied chemical reactions of the last half century. Turing was already famous for his seminal advances in mathematics, computer science, and cryptography. His single foray into chemistry, his paper on morphogenesis published in the Philosophical Transactions of the Royal Society, ${ }^{6}$ remains pivotal today in efforts to understand the role of symmetry breaking in the origin of life and in everyday living systems.

Indeed, much of the motivation for studies of nonlinear chemical systems stems from the notion that we can learn from them something about the dynamics of biological systems, which are generally much more complex and difficult to characterize. In chemistry, even complicated systems like the $\mathrm{BZ}$ reaction are relatively straightforward to characterize, as the state variables are typically simple concentrations of the dynamically significant chemical species, and the governing equations can be derived from the well understood rules of chemical kinetics coupled with transport processes such as diffusion and advection. In contrast, the state variables in biological systems are often challenging to identify, and characterizing the transport processes can be even more difficult. Hence, we hope that studies of relatively simple chemical systems such as the $\mathrm{BZ}$ reaction can offer insights into the surprisingly similar dynamics of biological systems. ${ }^{7}$

There are many fascinating stories about the early days of nonlinear chemical dynamics and the pioneers such as Turing, Belousov, and Zhabotinsky. One very early pioneer was Robert Luther, who succeeded Wilhelm Ostwald in the Physico-Chemical Institute at the University of Leipzig. Luther thought the propagating reaction-diffusion waves he had discovered might be relevant to signal transmission in neurons, and he presented his work in $1906 .{ }^{8-10}$ He proposed a formula for wave propagation velocity, $v=a(k D)^{1 / 2}$, where $a$ is a proportionality constant, $k$ is the rate constant, and $D$ is the diffusion coefficient, which is equivalent to the wave velocity equation published by Fisher ${ }^{11}$ and Kolmogorov et al. ${ }^{12}$ some 30 years later. He even presented a demonstration experiment of front propagation in the permanganate oxidation of oxalate-but in the audience, Walther Nernst was unconvinced. Nernst thought neuronal signal transmission is more like crystal growth, and in a surprisingly acrimonious debate, fully detailed in the proceedings, told Luther he would wait for his full publication [before believing it].

Many such stories and the science behind them can be found in a number of accounts and reviews. ${ }^{13-20} \mathrm{We}$ also 
note that there have been several Chaos Focus Issues devoted partly or entirely to nonlinear chemical dynamics. ${ }^{21-24}$ We describe some of the milestones below in our account of nonlinear chemical dynamics since the inception of Chaos-in celebration of its 25th anniversary.

\section{THE PAST 25 YEARS (AND A LITTLE BIT BEFORE)}

If we were to identify three themes that dominate the "early days" of nonlinear chemical dynamics, up to about 1990, they might be these:

(1) Chemical reactions with appropriate nonlinearities in their kinetics can give rise to such dynamical phenomena as multistability, periodic oscillation, and chaos.

(2) In spatially distributed systems, the interaction of diffusion with reaction kinetics leads to patterned behavior, including spirals and other traveling waves, Turing patterns, and spatiotemporal chaos.

(3) All of these phenomena are consistent with the laws of physics and chemistry, in particular, thermodynamics and kinetics, and an understanding of the general principles that govern these systems allows us both to construct reliable models and to design systems that exhibit behaviors of interest. The work of Prigogine and the Brussels school played a key role in helping chemists to appreciate the wealth of possibilities that become accessible when one studies systems far from equilibrium. $^{25}$

In this section, we describe briefly some of the most important developments of the past quarter century, beginning about 1990. Again, we can point to three distinguishing, and interacting themes:

(1) The spatial and temporal patterns identified and characterized in the earlier period can be manipulated, controlled, and transformed into one another by carefully chosen, relatively small external perturbations.

(2) In many systems, interesting behavior arises as the result of interactions between many subunits, each of which possesses a nonlinear dynamical behavior. The resulting "emergent" dynamical behavior can be considerably more complex than that of a single isolated subunit.

(3) Novel dynamical phenomena occur in "structured media" 16 consisting of spatially distributed arrays, which may be periodic or random, of distinct interacting reaction zones. Thus, for example, if one studies the BZ reaction in a microemulsion consisting of nanodroplets of water suspended in a continuous oil phase, the variety of patterns observed is far richer than what is seen for the same reaction in homogeneous aqueous solution.

\section{A. Control of chaos and other dynamical behavior}

The 1990s saw a flurry of theoretical and experimental activity on control and synchronization of chaos, and a Chaos Focus Issue was devoted to these topics. ${ }^{26}$ While work on control by goal dynamics had appeared earlier, ${ }^{27}$ the celebrated 1990 paper by Ott, Grebogi, and Yorke $(\mathrm{OGY}){ }^{28}$ which introduced a feedback control method that did not require knowledge of the governing equations, stimulated great interest in controlling chaos. Soon many "chaoticians" were using the OGY method or variants of it in attempts to stabilize unstable periodic orbits in their favorite chaotic system. The first successful application of these techniques in an experimental system, to our knowledge, was by Ditto, Rauseo, and Spano, with period- 1 and 2 stabilized in a chaotic magnetoelastic ribbon. ${ }^{29}$ Other experimental demonstrations of controlling chaos followed in a chaotic laser system, ${ }^{30}$ a thermal convection loop, ${ }^{31}$ and the Belousov-Zhabotinsky reaction. ${ }^{32}$ Perhaps the world's record for stabilizing high-periodicity orbits was attained by Hunt, ${ }^{33}$ who stabilized period-23 in a diode resonator.

Advances in controlling chaos led to a range of other applications of control theory in dynamical systems. Tracking unstable states is a natural extension of stabilizing them and is closely related to continuation methods such as AUTO, developed for bifurcation analysis of mathematical models. ${ }^{34}$ Early experiments included tracking unstable periodic orbits in a chaotic Duffing circuit, ${ }^{35}$ unstable stationary states in a chaotic multimode laser, ${ }^{36}$ and unstable periodic orbits in the chaotic BZ reaction. ${ }^{37}$ These and other studies established tracking methods as an effective method for bifurcation analysis of experimental systems. Tracking in spatiotemporal systems was also studied, including stabilizing and tracking unstable Turing patterns through mixedmode chaos in the Gray-Scott model. ${ }^{38}$

Many other applications of control theory to nonlinear systems, typically with simple linear control, were reported in the ensuing decade. The stabilization of an unstable state can be of great practical importance, for example, controlling a system that becomes dangerously chaotic, such as a chemical reactor. Control also can provide information for characterizing the dynamics of a system, as in the examples of tracking described above. Another example is the stabilization of unstable propagating waves. ${ }^{39,40}$ In a photosensitive BZ system, a propagating wave segment can be stabilized with a feedback algorithm that adjusts the excitability of the medium to yield a stable wave segment. This rather counterintuitive result offered a new perspective on the excitability boundary for spiral wave behavior in two-dimensional systems. The stable wave segment size as a function of excitability defines the boundary, above which spiral waves are supported and below which wave segments contract and disappear.

Once wave segments are stabilized, their motion can be readily controlled by feedback based on spatiotemporal excitability gradients. ${ }^{41,42}$ Thus, stabilized waves can be directed to propagate along any particular target trajectory with a light intensity gradient, within the limits of the response time of the system. Complex patterns of wave propagation are possible, as shown in Figure 1, as well as Brownian-like diffusive behavior with randomly varying excitability gradients. Similar propagating wave segments of depolarized tissue in the brain are observed in migraine episodes ${ }^{43,44}$ and it has been suggested that the feedback stabilizing these wave segments is self-sustaining, arising from the depolarized tissue itself. 


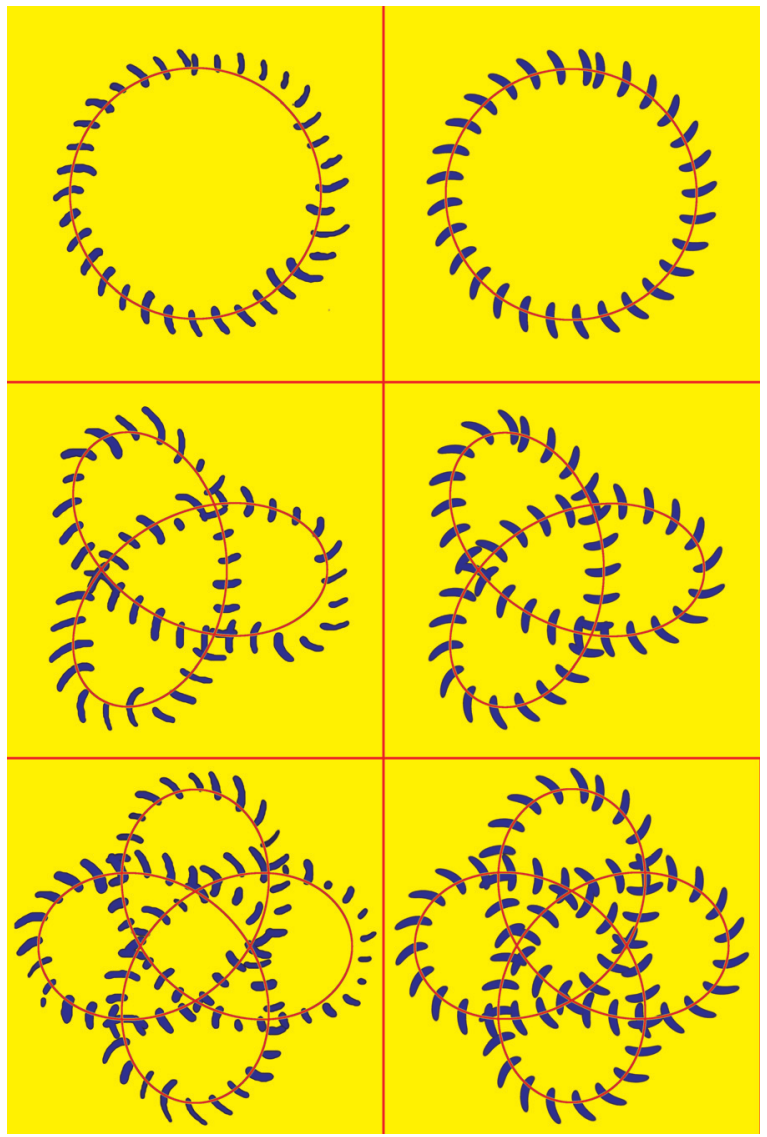

FIG. 1. Stabilized wave segments (blue) propagating along target trajectories (red) resulting from controlling propagation with light intensity gradients, experiments (left) and simulations (right). Reprinted with permission from T. Sakurai et al., Science 296, 2009-2012 (2002). Copyright 2002 AAAS.

\section{B. Spiral wave dynamics}

The first accounts of spiral waves in the BZ reaction were published, independently, in 1971 by Zhabotinsky and Winfree. ${ }^{45,46}$ Wave behavior in a two-dimensional medium, with concentric rings emanating from pacemaker sites, was described by Zaikin and Zhabotinsky in their seminal paper of $1970,{ }^{5}$ and Winfree followed with his landmark paper on spiral waves in $1972 .{ }^{47}$ Their discovery set into motion extensive experimental and theoretical studies that continue today.

One of the most remarkable aspects of spiral wavesother than the fact that they are fundamental self-sustaining sources of spatiotemporal order-is that these waves do not necessarily rotate rigidly around a circular "core," but more often display complex spiral tip trajectories. As early as 1973 , Winfree noted in his paper on scroll waves ${ }^{48}$ that the "spiral wave does not propagate quite in circles around a stationary center, but rather meanders in loops." In 1986, Winfree $^{49}$ published experiments and Oregonator ${ }^{50}$ simulations on complex spiral tip rotation. The experimental and computational evidence was extensive; however, he was not completely satisfied, because the Oregonator is not a quantitative model. Video imaging by Swinney and co-workers ${ }^{51}$ unequivocally showed that the spiral tip traces out hyperand hypocycloid trajectories, changing the number of lobes and from inward to outward as a bifurcation parameter such as concentration is varied. These beautifully intricate patterns spurred further theoretical studies, such as casting the dynamics into a normal form by Barkley et al. ${ }^{52}$

While early studies of spiral wave behavior were carried out in quasi-two dimensional thin films of BZ reaction mixtures, Winfree also began exploring waves in threedimensional media. ${ }^{48}$ The two-dimensional spiral wave represents a cross section of a three-dimensional scroll wave, in which the spiral core extends as a scroll filament. Scroll waves typically have been studied in a filament ring configuration, which arises from an experimental and computational protocol for generating these waves from expanding circular waves. Today, studies of scroll ring dynamics are facilitated by optical tomography, ${ }^{53,54}$ and a major topic of interest is negative filament tension giving rise to three-dimensional "Winfree turbulence." 55,56 These and other techniques have allowed a sophisticated view of scroll wave dynamics, including how scroll waves interact with boundaries ${ }^{57}$ and with each other. ${ }^{58}$

Spiral waves have also been studied on nonplanar surfaces and in heterogeneous media. Spiral waves on the surface of a catalyst-loaded bead ${ }^{59}$ in a catalyst-free BZ solution exhibit a spiral tip source near the north pole of the sphere and an apparent spiral wave sink at the south pole, arising from unequal frequencies of rotation of an initial counterrotating spiral pair. Theoretical and computational studies have demonstrated that the spiral source-sink occurs due to the topological constraints of the spherical geometry and a heterogeneous environment that leads to different spiral frequencies. ${ }^{60}$ In a system with heterogeneity deliberately produced by printing patterns of the BZ catalyst on polysulfone membranes, spontaneous spiral wave formation occurs, with the waves having geometrical shapes that depend on the catalyst pattern. ${ }^{61}$

Although spiral waves were first found in the BZ reaction, another important chemical reaction for studies of spatiotemporal dynamics has been the CO-Pt system, developed by Ertl and co-workers. ${ }^{62,63}$ The oxidation of $\mathrm{CO}$ by $\mathrm{O}_{2}$ on single-crystal $\mathrm{Pt}(110)$ occurs with surface reconstruction, which provides a source of feedback that leads to a rich variety of spatiotemporal patterns, including rotating spiral waves, target patterns, standing waves, and turbulence. ${ }^{64,65}$ The gas phase reactants at very low pressure minimize thermal effects and allow well defined and controllable global feedback. The spatiotemporal behavior can be monitored in real time using photoemission electron microscopy (PEEM), with a spatial resolution of about $1 \mu \mathrm{m} .{ }^{66,67}$ Modeling studies, which incorporate the global feedback and surface reconstruction, have been remarkably successful in describing the spatiotemporal dynamics of the CO-Pt system. ${ }^{6-70}$

\section{Patterns in structured media}

The first studies of nonlinear chemical dynamics almost all involved reactions in simple aqueous solution. Temporal oscillations, multistability, chaos, target patterns, and spiral waves were observed and characterized in beakers, flow reactors, and petri dishes through the mid-1980s. The introduction of the continuous flow stirred tank reactor (CSTR) 
into this field in the late $1970 \mathrm{~s}^{71}$ made it possible to maintain an oscillating reaction far from equilibrium and led to the first systematic design of new chemical oscillators ${ }^{72}$ as well as to the discovery of chaos in the BZ reaction. ${ }^{73}$

With growing interest in spatial patterns, it was clear by the late 1980s that a device that allowed for the inflow of fresh reactants into a spatially distributed system, a continuously fed unstirred reactor (CFUR), was essential. In 1988, the Austin group published the first results on sustained spiral waves in a CFUR consisting of a thin disk of chemically inert gel that hosted the reactant flows and suppressed convective motion. ${ }^{74} \mathrm{~A}$ few years later, the Bordeaux group solved a forty year old problem by using the chlorite-iodidemalonic acid (CIMA) reaction ${ }^{75}$ in a CFUR to produce the first experimental example ${ }^{76}$ of the temporally stationary, spatially periodic, reaction-diffusion patterns proposed by Turing $^{6}$ as a mechanism for morphogenesis in living systems. Gel reactors soon became a standard tool in nonlinear chemical dynamics, giving rise to a wealth of novel behaviors including spatiotemporal chaos $^{77}$ and spatial bistability. ${ }^{78}$

Like the discovery of the first chemical oscillators, finding Turing patterns involved a considerable component of serendipity. As Turing showed in his seminal paper, ${ }^{6}$ in an activator-inhibitor system with appropriate chemical kinetics, the inhibitor must diffuse significantly more rapidly than the activator in order for stationary patterns to arise. This condition poses an insuperable problem in water, where the diffusion coefficients of nearly all soluble species lie within a factor of two to three of one another. This accounts for the forty year time lag between Turing's prediction and its experimental realization. The necessary difference in diffusion rates arose in the CIMA system from the fact, which was unanticipated by the researchers, that the starch used as an indicator to visualize the concentration of the iodinecontaining activator species becomes trapped in the pores of the gel. The activator molecules form a reversible complex with the immobilized starch, and their effective diffusion rate is reduced by a factor equal to the fraction of time they are trapped in the complex. This rate can be tuned into a range that supports Turing patterns either by a lucky choice or deliberately, once one understands the underlying mechanism. ${ }^{79}$ This understanding leads to a general approach to designing Turing patterns based on the idea of slowing down the diffusion of the activator by introducing a complexing agent of considerably smaller (or zero) mobility. ${ }^{80}$ Subsequently, an alternative design procedure based on spatial bistability was proposed and implemented to generate Turing patterns in the thiourea-iodate-sulfite reaction. ${ }^{81}$

Almost from the time of its discovery, the BZ reaction has been the cornerstone of nonlinear chemical dynamics. Nearly every phenomenon of importance was either discovered or investigated in detail in this prototype system, with one glaring exception-Turing patterns. The BZ's activator and inhibitor species insist on diffusing at similar rates, and it has proved impossible (at least so far) to find a complexing agent to slow down the activator (or speed up the inhibitor). Finally, in 2001, a new kind of structured medium, a reverse microemulsion, made it possible to generate Turing patterns in the BZ reaction. ${ }^{82}$ The system, referred to as BZ-AOT, contains a mixture of oil (octane), water, and a surfactant, sodium bis(2-ethylhexyl) sulfosuccinate, known as AOT. In appropriate proportions, the mixture forms reverse micelles-nanodroplets of water surrounded by a monolayer of AOT floating in the continuous oil phase. The droplets are nearly monodisperse, and their mean size and spacing can be controlled by varying the proportions of oil, water, and AOT. ${ }^{83}$ Since all the BZ reactants are polar, if one introduces them into an AOT reverse microemulsion, they partition into the water droplets, each of which becomes a tiny reactor undergoing the $\mathrm{BZ}$ oscillating reaction. The droplets interact with each other via two distinct mechanisms. First, they undergo Brownian motion, colliding with one another, momentarily coalescing, and then redispersing after mixing their contents. Second, the reaction produces nonpolar intermediates, which can enter the oil, diffuse through it, and on occasion enter a new droplet. The latter process is significantly faster than the Brownian motion of entire droplets, since it involves single molecules rather than aggregates containing typically tens of thousands of molecules. It turns out that the major nonpolar intermediate is bromine, which acts as an inhibitor of the $\mathrm{BZ}$ reaction, so the Turing condition of long range inhibition and short range activation can be satisfied. In Figure 2, we show three-dimensional Turing patterns in the BZ-AOT system, ${ }^{84}$ obtained using a tomographic method originally used to study three-dimensional scroll rings in the $\mathrm{BZ}$ reaction. ${ }^{85}$ By varying the composition of the

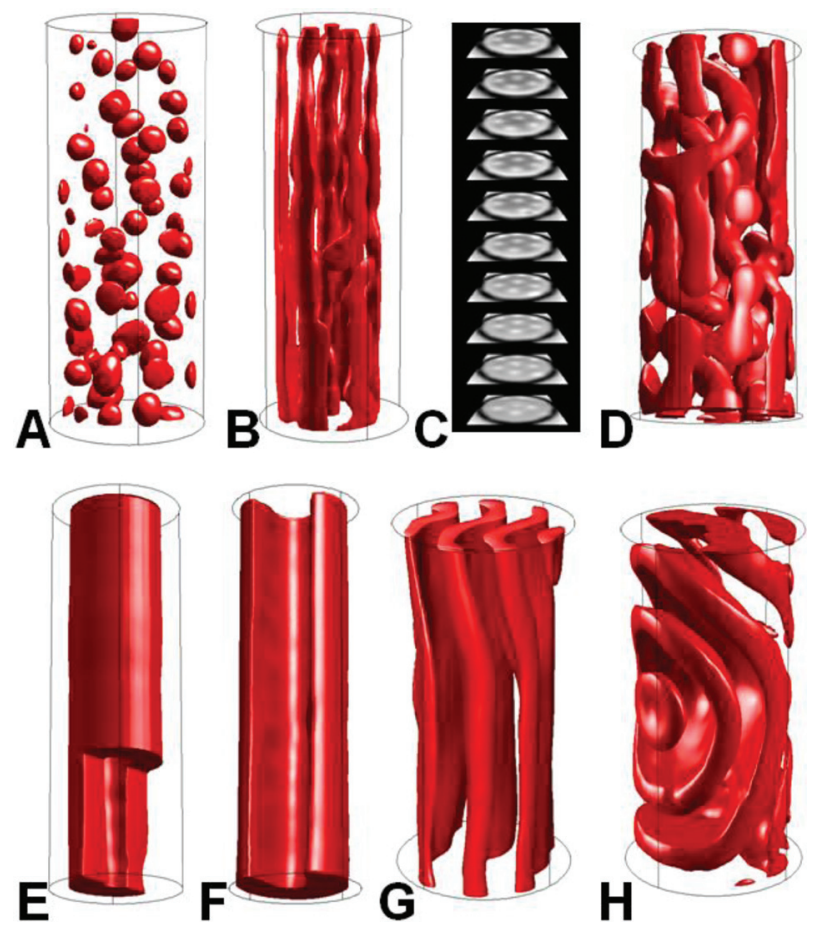

FIG. 2. Tomographically reconstructed concentration fields for 3D Turing patterns. Spots (A), hexagonal close-packed rods (B), horizontal crosssections (C) taken in the vertical direction through data array in (B), labyrinthine pattern (D), tube (E), half-pipe (F), lamellar pattern $(\mathrm{G})$, and concentric hemispherical lamellae $(\mathrm{H})$. Inner diameter of capillary is $0.6 \mathrm{~mm}$ for (A)-(D), (G), and (H) and $0.3 \mathrm{~mm}$ for $(\mathrm{E})$ and $(\mathrm{F})$. A short segment at the bottom of the front half of pattern (E) has been removed to reveal the inner structure. Reprinted with permission from Bánsági et al., Science 331, 1309-1312 (2011). Copyright 2011 AAAS. 
microemulsion and the concentrations of the $\mathrm{BZ}$ species, one can find a remarkably rich set of dynamical phenomena in addition to Turing patterns in the BZ-AOT system. These include antispirals,${ }^{86}$ which, unlike the spiral waves found in aqueous solution, travel toward rather than away from the spiral center; segmented waves, both plane ${ }^{87}$ and spiral, ${ }^{88}$ in which waves break up transverse to their direction of propagation into coherently moving fragments; accelerating waves ${ }^{82}$ which unlike normal $\mathrm{BZ}$ waves, speed up before colliding and then propagate away at right angles after collision instead of annihilating; and oscillons, ${ }^{89}$ localized regions of oscillatory behavior. Figure 3 gives a sense of the wide range of behavior observed as one traverses the BZAOT parameter space.

Microemulsions open up a widow into an abundant variety of novel patterns, but, because each droplet is only about $10 \mathrm{~nm}$ in diameter, one can neither monitor nor control the behavior of individual drops. Microfluidics offers another experimental approach to studying nonlinear phenomena in reacting systems. One of the first uses of this powerful technique in nonlinear chemical dynamics was Ismagilov's design of a simple model of blood coagulation using an autocatalytic reaction system in a capillary tube. ${ }^{90}$ More recent efforts have used microfluidics to produce one- and twodimensional arrays of aqueous droplets, typically $50-200 \mu \mathrm{m}$ in diameter and separated by an oil drop of similar dimensions. Droplets of this size can remain stationary in a capillary tube, can be observed through a microscope, and, when a photosensitive version of the $\mathrm{BZ}$ reaction is introduced into the water droplets, can be controlled by illuminating selected drops to suppress oscillation. Studies of one-dimensional drop arrays show synchronization, most often antiphase, ${ }^{91}$ because interdrop coupling through inhibitory bromine is dominant, though more complex patterns are also observed. ${ }^{92}$ In two dimensions, drops spontaneously organize into hexagonal arrays, where a variety of patterns, some stationary, some oscillatory, and some mixed, have been characterized. ${ }^{93}$ Figure 4 shows a remarkable example of such a study, ${ }^{94}$ in which a two-dimensional array of microfluidically generated BZ drops first undergoes "chemical

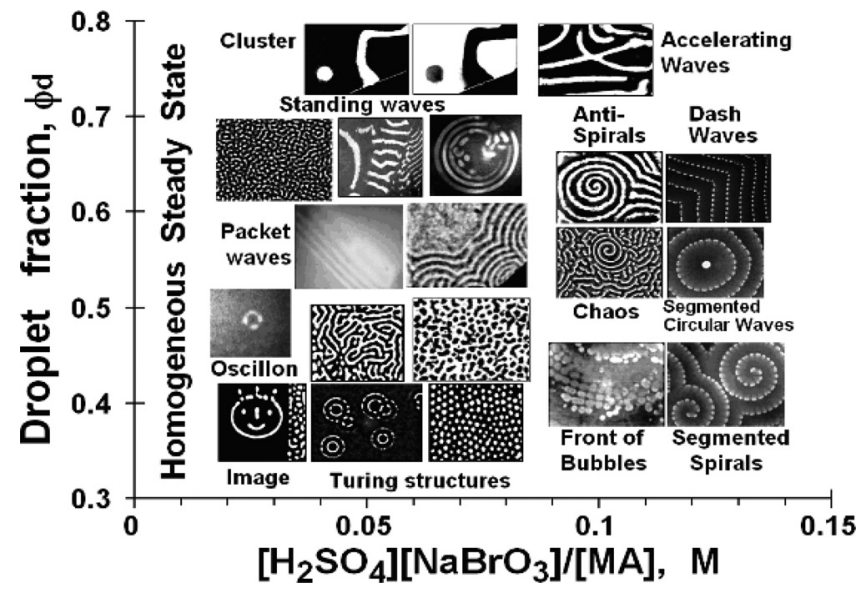

FIG. 3. Overview of pattern formation in the BZ-AOT system as the compositions of the microemulsion and the $\mathrm{BZ}$ reactants are varied. $\phi_{\mathrm{d}}$ is the fraction of the total micro-emulsion volume occupied by the droplets. differentiation" to a Turing-like pattern in which some drops are in a reduced stationary state while others are in an oxidized state. All drops have the same radius at this stage. A bit later, apparently as a result of osmosis - the reduced and oxidized states have different concentrations of ions-the drops experience "physical differentiation," with the oxidized drops shrinking and the reduced drops swelling. Turing, in fact, suggested osmotic effects as a possible origin of morphogenesis in his paper over sixty years earlier ${ }^{6}$ !

\section{Materials and chemomechanical transduction}

Many of the most important practical results of chemical research have involved the creation and understanding of materials with novel and useful properties, whether for clothing, construction, medical, or other applications. Over the past twenty-five years, nonlinear chemical dynamics has moved in the direction of applying its capabilities to problems in materials science. Polymers, in particular, have been the subject of detailed investigation. ${ }^{95}$ One of the most notable advances has been the development of frontal polymerization, ${ }^{96}$ in which monomers are converted to polymer in a local reaction zone that propagates through the system because of thermal acceleration of the rate as the polymer is formed and/or due to the gel effect, i.e., the decrease in the termination rate due to the increased viscosity in the polymerization region. Many dynamic instabilities have been observed and characterized in frontal polymerization systems, ${ }^{97}$ and the phenomenon has been exploited to produce functionally gradient materials ${ }^{97}$ as well as fast-curing adhesives and resins. ${ }^{98}$

A key step in, literally, linking polymers to nonlinear chemical dynamics was taken by Yoshida, ${ }^{99}$ who succeeded in covalently bonding the BZ catalyst, ruthenium tris-bipyridyl (Ru(bipy) $)_{3}$ ) to poly(N-isopropylacrylamide) (PNIPAAm). Gels formed from PNIPAAm undergo a sharp volume change when they pass through their lower critical solution temperature (LCST) due to an uptake or loss of water. This same volume change takes place isothermally in response to changes in the redox state of $\mathrm{Ru}(\text { bipy })_{3}$ attached to PNIPAAm, if the gel is placed in a solution containing the BZ reactants. The temporal concentration oscillations of the BZ reaction are thus transformed into mechanical (volume) oscillations of the gel. Yoshida has suggested a number of clever applications of such gels, including ciliary actuators ${ }^{100}$ and drug delivery modules. ${ }^{101}$ The chemomechanical transduction observed in BZ gels strongly resembles the behavior of muscle tissue in converting the chemical energy stored in ATP (adenosine triphosphate) into mechanical motion.

Balazs $^{102}$ developed a computational framework that combines the Oregonator model's description of the BZ chemistry with the mechanical forces arising from the fluid movement in the Yoshida gel as its chemical composition varies in time. With additional terms to describe the photoreaction of the catalyst, this "gel lattice spring model" predicts that a strip of gel will behave as a photophobic "chemical worm," moving away from more brightly illuminated regions, ${ }^{103}$ as shown in Figure 5. Recent experiments ${ }^{104}$ 

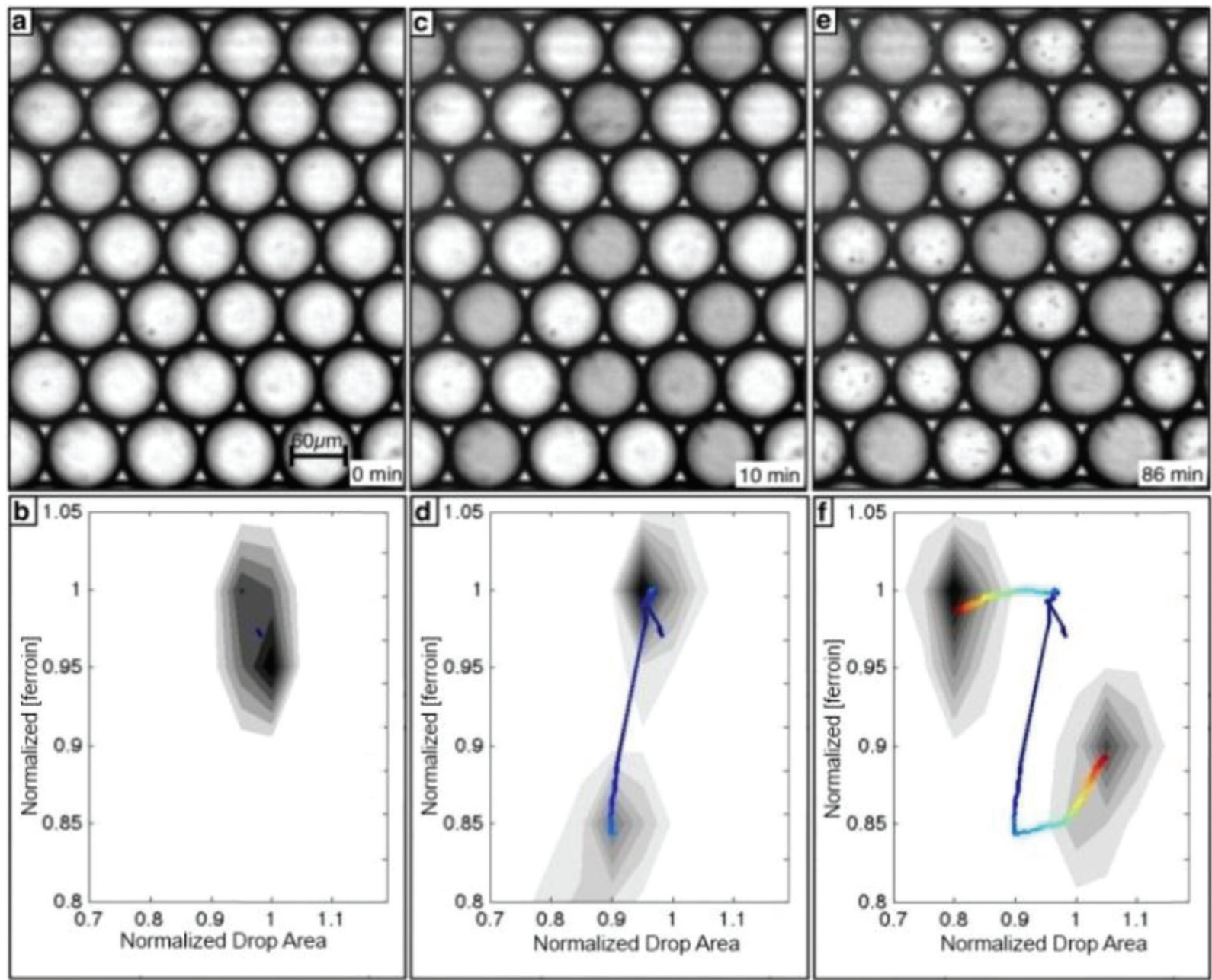

$\begin{array}{llllllllll}0 & 10 & 20 & 30 & 40 & 50 & 60 & 70 & 80 & \text { Time }(\mathrm{min})\end{array}$

FIG. 4. Images (upper row) and histograms (lower row) of drops demonstrating morphogenesis. Histograms show fraction of original concentration of reduced BZ catalyst $v s$. fraction of original drop area. Gray scale in histograms is proportional to the number of drops with a given area and intensity. Color-coded lines track the center of each peak as a function of time. (a) and (b) Initially, drops are nearly homogeneous in both intensity and size. (c) and (d) At intermediate times, drops undergo a Turing bifurcation, becoming heterogeneous in oxidation state, but remaining homogeneous in size, as seen by the differentiation into lighter and darker drops. (e) and (f) Later, drops become heterogeneous in both oxidation state and size. The oxidized (brighter) drops shrink and the reduced drops swell. Reprinted with permission from Tompkins et al., Proc. Natl. Acad. Sci. 111, 4397-4402 (2014). Copyright 2014 National Academy of Sciences.

have confirmed this prediction and have revealed a regime of phototropic behavior as well, as shown in Figure 6.

The prospects for tailoring the properties of oscillatory gels are bright, as the power of synthetic chemistry has only begun to be applied to systems of this sort. Recent efforts have demonstrated, for example, that by modifying the ligands attached to the ruthenium catalyst one can create gels in which the now multidentate catalyst also serves as the crosslinker for the gel, creating a fully three-dimensional architecture, as opposed to the lower-dimensional structure of the Yoshida gel, in which the catalyst is simply pendant on the polymer chain and an additional small molecule must be introduced as the crosslinker. ${ }^{105}$

\section{E. Synchronization: Collective behavior and emergence}

One of the most active areas in complex systems research is focused on the synchronization of interacting dynamical subsystems. Again, the impetus is largely from biology, where synchronization is pervasive and plays a vital role in such fundamental processes as metabolism, peristalsis, and the rhythmic beating of the heart. The synchronization of coupled oscillators is the most important paradigm for fundamental studies of these and other complex processes.

An early synchronization study by the biologists Aldridge and Pye ${ }^{106}$ focused on the behavior of yeast cells in stirred suspensions as a function of cell density, monitoring NADH (nicotinamide adenine dinucleotide) fluorescence. They found the yeast cells exhibited synchronized oscillations above a critical cell density, but below this density, only a quiescent global signal was observed. It was clear that the yeast cells were communicating above the critical density, and this study established the yeast cell suspension as a model system for inter-cellular signaling. What was not clear was the nature of the transition between synchronized oscillations above the critical density and the quiescent global signal at lower densities. Around 30 years after the Aldridge 
a)

)

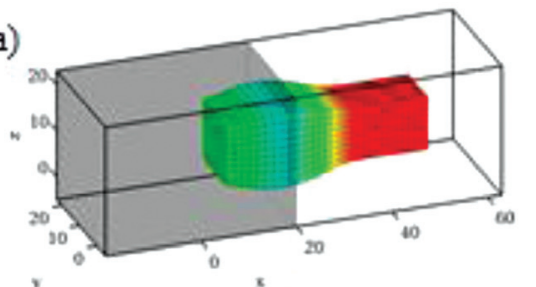

b)

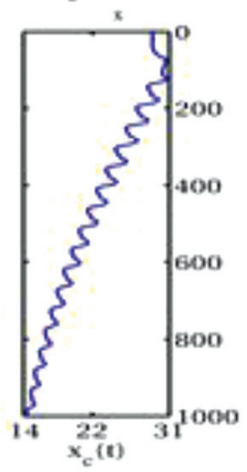

c)

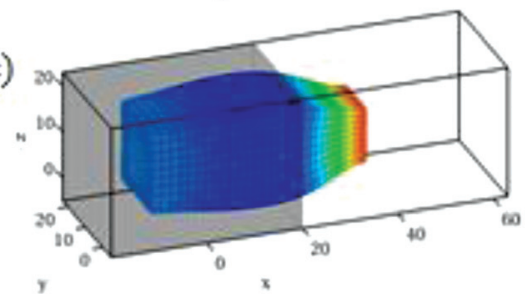

d)

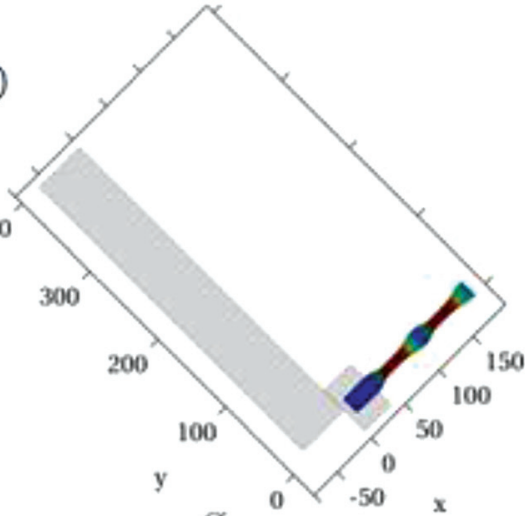

e)

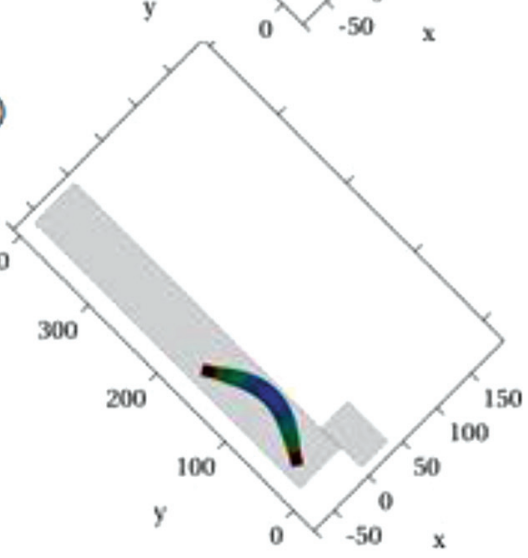

FIG. 5. Simulation of a phototactic "chemical worm." Gray indicates the non-illuminated regions. (a) and (c) Early- and late-time positions, respectively, of the BZ worm; (b) evolution of the $\mathrm{x}$ coordinate of the gel's center; (d) and (e) reveal how the worm makes a turn. The velocity of these millimetersized worms is approximately $1.2 \mu \mathrm{m} / \mathrm{s}$. Reprinted with permission from Dayal et al., Langmuir 25, 4298-4301 (2009). Copyright 2009 American Chemical Society. and Pye results, De Monte et al. ${ }^{107}$ studied the same yeast system, but now in a flow reactor that allowed stationary states to be established. They found on perturbing the system below the critical density, where the system is quiescent, that an exponential decay to a focus stationary state is exhibited. This provided strong evidence that the transition is not like a
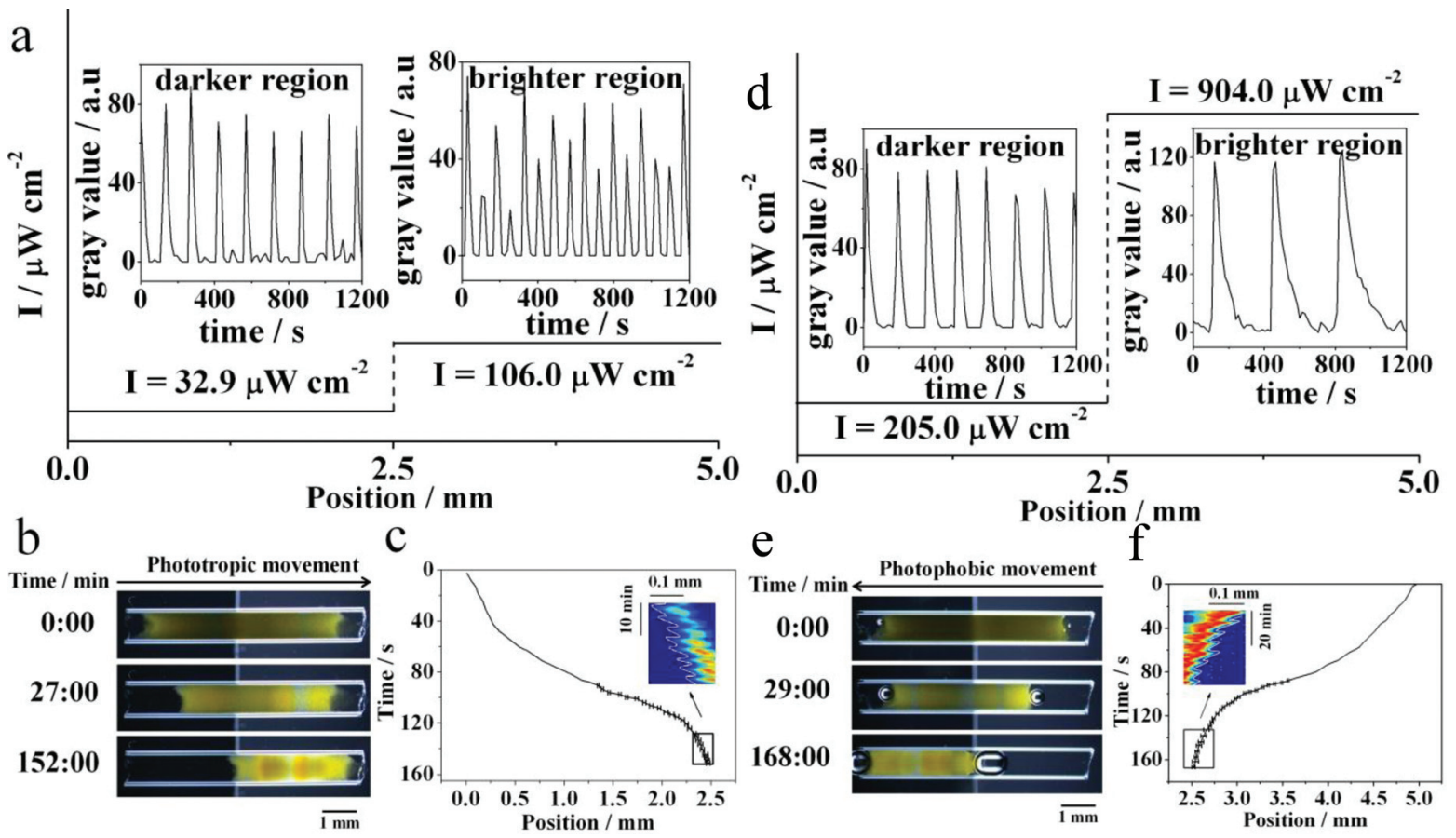

FIG. 6. Experiments demonstrating phototropic and photophobic movement of a BZ photosensitive gel (yellow) within a capillary tube under spatially nonuniform illumination. (a) and (d) Distribution of illumination and local dynamics (gray levels proportional to $\left[\mathrm{Ru}(\mathrm{bpy}) 3^{3+}\right]$ ) at central points of the two illuminated regions. (b) and (e) Snapshots of gel movement. Location of right (c) or left (f) edge of the gel vs. time. Reproduced with permission from Lu et al., Chem. Commun. 49, 7690-7692 (2013). Copyright 2013 The Royal Society of Chemistry (RSC). 
synchronization-desynchronization transition but rather one in which each oscillator changes from oscillatory to steady state behavior, much like a quorum sensing transition. ${ }^{108}$

A stirred suspension of individual chemical oscillators, coupled by the intermediate species they produce and transfer to the solution, provided surprising insights into the synchronization transitions of the yeast system. ${ }^{109}$ Image analysis, as shown in Figure 7, of catalyst-loaded particles in catalyst-free BZ solutions revealed two types of synchronization transitions. One was much like the quorum sensing transition observed in yeast cell suspensions, ${ }^{107}$ and the other was a gradual transition from unsynchronized to synchronized oscillations. Hence, the BZ oscillator system showed that both types of synchronization transitions are possible and how these transitions are related. It seems likely that oscillatory microorganisms such as yeast and some bacteria will also exhibit both transitions if these dynamical behaviors lie in accessible parameter ranges.

As nonlinear dynamics developed in physics and chemistry, usually with an eye on the naturally nonlinear systems of biology, attention turned toward collective behavior. Popular images of swarming starlings and schooling fish fascinated not only the general public but nonlinear dynamicists as well. A challenge for chemists was to find collective behavior in nonliving systems with features like those seen in living systems. This challenge has been met in the recent experimental development of micro- and nanoscale selfpropelled particles, which have motility features resembling those seen in bacteria and algae.

The first examples of chemically powered self-propelled particles were Ag-Pt rods in $\mathrm{H}_{2} \mathrm{O}_{2}$ solutions, in research pioneered by the groups of Sen and Mallouk. ${ }^{110-113} \mathrm{The} \mathrm{Ag}-\mathrm{Pt}$ rods propel themselves by an electrophoretic mechanism, where the oxidative decomposition of $\mathrm{H}_{2} \mathrm{O}_{2}$ occurs at the $\mathrm{Pt}$ end, and the reductive decomposition of $\mathrm{H}_{2} \mathrm{O}_{2}$ occurs at the

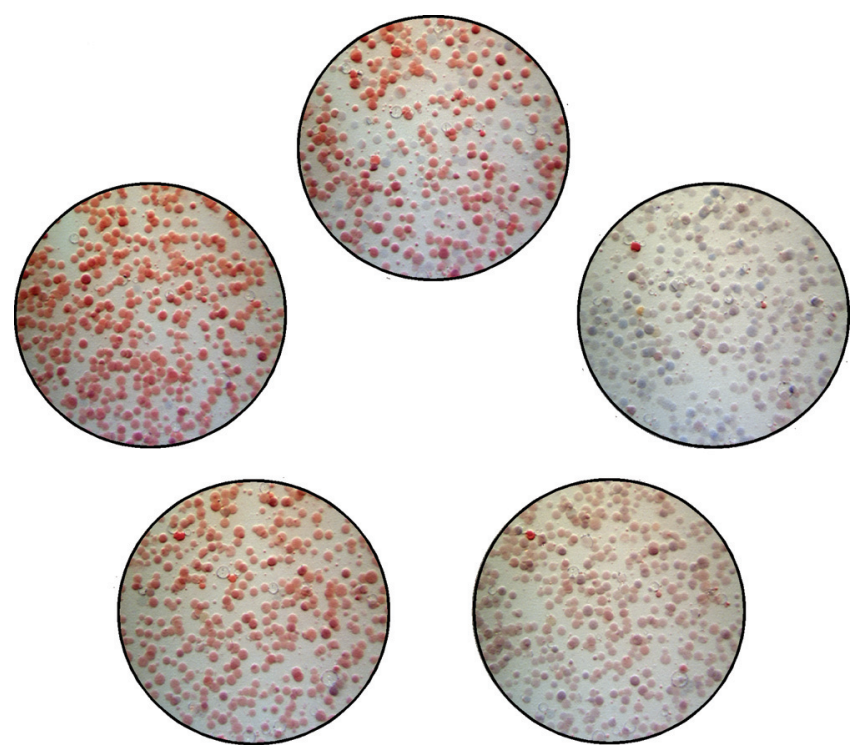

FIG. 7. Images of synchronized BZ oscillators in a stirred suspension of $\sim 100000$ catalyst-loaded beads in a catalyst-free solution. Reprinted with permission from Taylor et al., Science 323, 614-617 (2009). Copyright 2009 AAAS.
Au end, with electrical conduction through the metallic rod. A flow of $\mathrm{H}^{+}$occurs, maintaining charge balance, and the resulting electrophoretic motion propels the particle. Several new types of self-propelled particle systems followed, including Pt-polystyrene and Pt-silica Janus particles in $\mathrm{H}_{2} \mathrm{O}_{2}$ solution, ${ }^{114,115}$ which rely on diffusiophoresis for propulsion. The catalytic decomposition of $\mathrm{H}_{2} \mathrm{O}_{2}$ at the Pt half of the particle results in a self-sustaining gradient of $\mathrm{O}_{2}$, which gives rise to the propulsion. ${ }^{116}$ Typical propulsive behavior can be seen in Figure 8, where the composite image shows the particle traveling generally in the same direction over a number of subsequent image frames, only to be interrupted by occasional large directional changes. ${ }^{115}$

While significant progress has been made in developing and characterizing individual self-propelled particles, observing collective behavior among populations of these particles has remained elusive. However, "molecule-like" particle agglomerates display fascinating and complex selfpropelled behavior, depending on the relative orientation of each particle in the group. ${ }^{117,118}$ A particularly compelling example of collective behavior has been observed in "living crystals" made up of micron size hematite cubes that are asymmetrically embedded in TPM (3-methacryloxypropyl trimethoxysilane) polymer spheres. ${ }^{119}$ When irradiated with 430-490 nm light in basic $\mathrm{H}_{2} \mathrm{O}_{2}$ solution, "living crystals" of around 35 particles form, with continually rearranging domains and self-propulsion.

Synchronization of macroscale chemical oscillators has played a major role in nonlinear chemical dynamics almost since its beginning. Flow reactors, for example, may be coupled via mass flow, electrochemically or through a variety of computer-mediated approaches. ${ }^{18}$ In most experiments, the coupling is continuous and symmetric, i.e., it resembles diffusion. Coupling between neural oscillators, in contrast, is generally pulsatile and asymmetric. A signal is transmitted from the presynaptic to the postsynaptic neuron

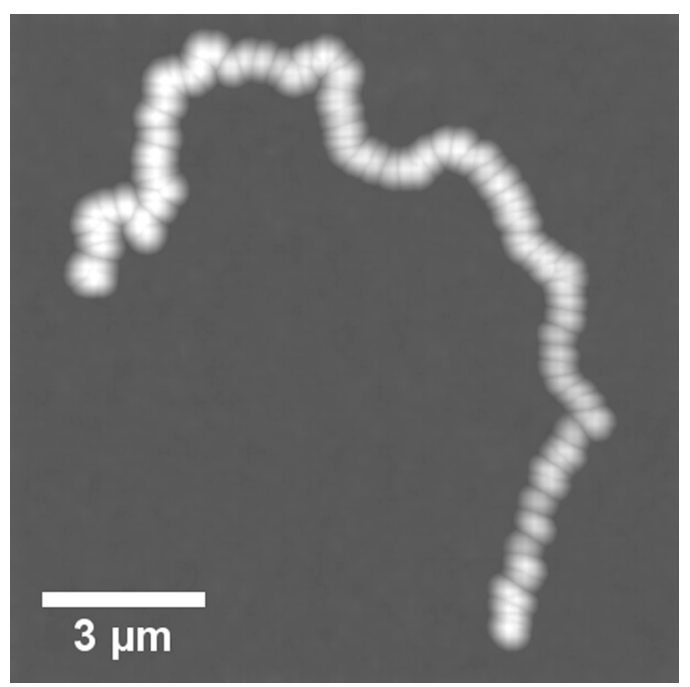

FIG. 8. Overlay images of a self-propelled Pt-silica particle in $10.0 \% \mathrm{H}_{2} \mathrm{O}_{2}$ taken at a rate of $\sim 56$ frames/s. Reprinted with permission from Ke et al., J. Phys. Chem. A 114, 5462-5467 (2010). Copyright 2010 American Chemical Society. 
only when the presynaptic cell's membrane potential passes a threshold. Recent experiments have shown how such behavior may be mimicked in coupled CSTRs containing the $\mathrm{BZ}$ reaction. ${ }^{120}$

\section{F. Synchronization: Chimeras and networks}

Recently, extensive research activity has focused on a surprising dynamical behavior in which synchronized and unsynchronized oscillators coexist. In 2002, Kuramoto and Battogtokh ${ }^{121}$ reported that a population of identical phase oscillators, coupled to each other in an identical manner, separates into subpopulations of synchronized and unsynchronized oscillators. Abrams and Strogatz ${ }^{122}$ aptly named this behavior a chimera, after the Greek mythological creature made up of a lion, goat, and serpent-animals normal as individuals but incongruous together, much like synchronized and unsynchronized oscillators. Shima and Kuramoto ${ }^{123}$ then reported a spectacular variation of this behavior in which a spiral wave in a two-dimensional array of oscillators rotates around a core of unsynchronized oscillators. The counterintuitive behavior of the chimera generated great interest, and many theoretical studies followed, with bifurcation analysis demonstrating the stability of this new type of dynamical state, ${ }^{122,124-126}$ and studies with nonlinear coupling, ${ }^{127}$ time delay, ${ }^{128}$ network configurations, ${ }^{129}$ and transient chaotic dynamics. ${ }^{130}$

Experimental studies with an electro-optical device ${ }^{131}$ and photochemically coupled BZ oscillators ${ }^{132}$ showed that similar chimera behavior could be found in the laboratory, where the oscillators were identically coupled but not necessarily identical. The BZ oscillator experiment followed the minimal model system of Abrams et al., ${ }^{125}$ where two groups of oscillators are globally coupled such that the oscillators within a group are coupled more strongly than the oscillators between the groups. The photochemical coupling relies on the photoexcitation of the $\mathrm{Ru}(\mathrm{bpy})_{3}{ }^{2+} \mathrm{BZ}$ catalyst, which produces the autocatalyst $\mathrm{HBrO}_{2}$ and typically leads to a phase advance of an oscillator. ${ }^{133,134}$ The coupled oscillator behavior, shown in Figure 9, includes the expected out-of-phase synchronization and phase clusters, and the unexpected-the chimera with coexisting synchronized and unsynchronized oscillators.

A number of experimental studies have now appeared. Examples of chimeras have been found in electrochemical systems, both with coupled individual oscillators ${ }^{135,136}$ and in a continuous medium with nonlinear global coupling. ${ }^{137,138}$ A chimera experiment with nonlocal coupling in a ring configuration has been carried out with photochemically coupled BZ oscillators. ${ }^{139}$ An intriguing mechanical experiment, based on two groups of 20 coupled metronomes on swings, with the swings coupled to each other by a spring, demonstrated robust chimera behavior. ${ }^{140}$

\section{A SYSTEMS APPROACH}

In recent years, the field of systems biology has grown rapidly and has drawn growing attention in both scientific and popular media. The complexity of living systems makes them natural candidates for a "systems approach," which
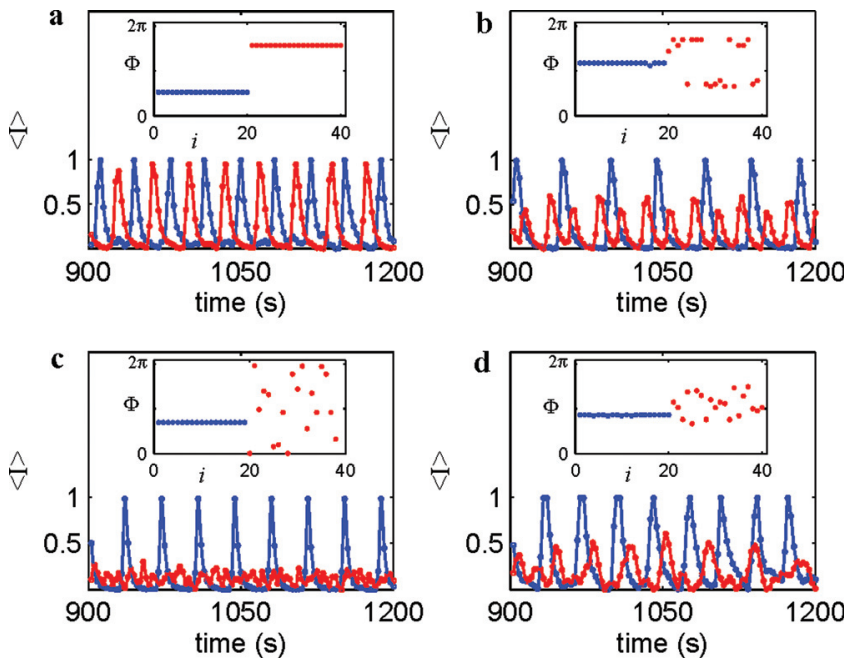

FIG. 9. Population of coupled chemical oscillators exhibiting two subpopulations (red and blue), which are synchronized out of phase (a), synchronized with one and two phase-cluster states (b), synchronized and unsynchronized-the chimera, and semi-synchronized, with occasionally bunching phase clusters. Insets show snapshots of the phase of each oscillator in the two groups. Reprinted with permission from Tinsley et al., Nat. Phys. 8, 662-665 (2012). Copyright 2012 Nature Publishing Group.

takes a multidisciplinary, non-reductionist, quantitative point of view and focuses on emergent properties that result from the interactions between large numbers of components. Systems biology has become the subject of books, journals, and even academic departments and institutes.

One might argue that before there was systems biology, there was systems physics, systems chemistry, systems etc. In fact, during the early days of systems biology, a biologist colleague remarked to one of the authors of this article, "You chemists have been doing this sort of thing for years, but just did not bother to call it systems chemistry." Physicists have probably been in the systems business the longest, going back, one might argue to Huygens' experiments in 1665 on the synchronization of pendulum clocks. ${ }^{141}$ We do not seek to address the issue of priority here, but rather to point out the many features shared by the biological, chemical, and physical phenomena to which a systems approach has been applied. These features have, in fact, provided much of the subject matter of Chaos over the past twenty-five years.

It seems fair to say, that many of the ideas and topics that characterize systems chemistry, now a subject in its own right, ${ }^{142}$ link that field closely with nonlinear chemical dynamics-complex, interacting, far-from-equilibrium systems. It should also be acknowledged that ideas from other "systems fields," particularly physics and biology, have spurred developments in chemistry, and perhaps there has been a bit of cross-fertilization in the other direction as well.

Several of the studies described in Secs. II A-IIF demonstrate what one might call a systems approach to nonlinear chemical dynamics-efforts to control chaos and other dynamical behavior, investigations of the interaction between chemical and mechanical phenomena, experiments and modeling of global feedback, discoveries of collective behavior 
such as quorum sensing, and studies of networks of coupled oscillators from the nano to the macro scale.

\section{PREDICTING THE FUTURE}

Niels Bohr is said to have remarked ${ }^{143}$ that "Prediction is very difficult, especially if it's about the future." Nonetheless, in an article of this sort, we feel obligated to at least speculate briefly on where we see nonlinear dynamics heading over the next quarter century. We believe that the field has reached a level of maturity and a breadth of scope that will enable its practitioners to focus their future efforts on problems of increasing practical importance and relevance to other areas of science and technology. Many of the trends that we anticipate are already becoming visible.

We expect that nonlinear chemical dynamics will continue to expand its reach beyond reaction and diffusion to explore systems involving mechanical forces, convection, advection, osmosis, and other transport phenomena. Understanding and controlling such systems have the potential to lead to new approaches to drug delivery, sensors, actuators, and other devices.

We expect continued progress in studying increasingly complex systems composed of multiple interacting parts, whether these are groups of reactions like metabolic pathways or discrete physical units like catalyst-loaded beads or the nanodroplets of a microemulsion. On the one hand, the tools developed over the past quarter century for analyzing networks have been relatively underappreciated by chemists and offer much promise for future application. On the other, the apparatus of statistical mechanics, developed two hundred fifty years ago, offers similar promise in dealing with aggregates consisting of large numbers of identical or similar subunits, each containing relatively few molecules, so that fluctuations become large. A typical BZ-AOT microemulsion, for example, contains about $10^{17}$ nanodroplets, each populated by perhaps a few hundred molecules of the key reactants.

The study of traditional chemistry can be largely divided into two areas-synthesis, i.e., designing and making molecules and materials, and analysis, i.e., establishing the molecular structure and composition of those molecules and materials. In nonlinear chemical dynamics, as in traditional chemistry, analysis came first. Scientists needed to understand the nature and properties of chemical substances before they could begin to construct them from simpler components. Nonlinear chemical dynamics has followed a similar path. Early efforts were devoted to describing such phenomena as oscillations, waves, and chaos (which had typically been discovered accidentally) and to making models that could simulate those behaviors. More recently, we have learned how to control and design chemical systems that display novel dynamic phenomena. We anticipate that this "synthetic" approach will grow in importance in the years to come.

In a sense, life itself is the preeminent example of nonlinear chemical dynamics. It involves chemical reactions operating far from equilibrium in open systems consisting of units that are physically structured and dynamically linked so as to generate such nonlinear phenomena as multistability, oscillations, chaos, spatial patterns, and waves. The Malthusian population explosion is nothing more than a statement that reproduction is an autocatalytic process. We expect that practitioners of nonlinear chemical dynamics will be increasingly drawn to explaining biological phenomena and to designing biomimetic systems, devices, and materials. The successes we have noted above in demonstrating such "bio"-phenomena as motility, quorum sensing, synchronization, chemomechanical transduction, and phototaxis should provide strong inspiration to those seeking to pursue this direction.

A particularly compelling problem, in which nonlinear chemical dynamics is already playing a growing role, is the origin of life. For example, a recent meeting ${ }^{144}$ sponsored by the European Union on Systems Chemistry marked the start of an initiative on "Emergence and Evolution of Complex Chemical Systems" that brings together researchers interested in subjects ranging from synthetic organic chemistry to hydrodynamics to mathematical modeling. Much of the focus of the meeting was on insights into the possible origin of life. We expect such wide-ranging meetings and collaborations to become common in nonlinear chemical dynamics in the years to come.

\section{ACKNOWLEDGMENTS}

I.R.E. thanks the National Science Foundation (Grant No. CHE-1362477) and the W.M. Keck Foundation for support of this research. K.S. thanks the National Science Foundation (Grant No. CHE-1212558).

${ }^{1}$ A. T. Winfree, "The prehistory of the Belousov-Zhabotinsky oscillator," J. Chem. Educ. 61, 661-663 (1984).

${ }^{2}$ B. P. Belousov, "A periodic reaction and its mechanism," Sbornik Referatov po Radiatsionni Meditsine (Med. Publ., Moscow, 1959), p. 145.

${ }^{3}$ A. M. Zhabotinsky, "A history of chemical oscillations and waves," Chaos 1, 379-386 (1991).

${ }^{4}$ A. M. Zhabotinsky, "Periodic kinetics of oxidation of malonic acid in solution (study of the Belousov reaction kinetics)," Biofizika 9, 306-311 (1964).

${ }^{5}$ A. N. Zaikin and A. M. Zhabotinsky, "Concentration wave propagation in two-dimensional liquid-phase self-oscillating system," Nature 225, 535-537 (1970).

${ }^{6} \mathrm{~A}$. M. Turing, "The chemical basis of morphogenesis," Philos. Trans. R. Soc. London, Ser. B 237, 37-72 (1952).

${ }^{7}$ V. K. Vanag and I. R. Epstein, "Pattern formation mechanisms in reaction-diffusion systems," Int. J. Dev. Biol. 53, 673-681 (2009).

${ }^{8}$ R. Luther, "Propagation of chemical reactions in space," J. Chem. Educ. 64, 740-742 (1987).

${ }^{9}$ K. Showalter and J. J. Tyson, "Luther's 1906 discovery and analysis of chemical waves," J. Chem. Educ. 66, 742-744 (1987).

${ }^{10} \mathrm{R}$. Luther, "Space propagation of chemical reactions," Z. Elektrochem. Angew. Phys. Chem. 12, 596-600 (1906).

${ }^{11}$ R. A. Fisher, "The wave of advance of advantageous genes," Ann. Eugen. 7, 355-369 (1937).

${ }^{12}$ A. Kolmogorov, I. Petrovskii, and N. Pisocunov, "A study of the diffusion equation with increase in the amount of substance, and its application to a biological problem," Byul. Moskovskogo Gos. Univ. 1, 1-25 (1937).

${ }^{13}$ F. Sagués and I. R. Epstein, "Nonlinear chemical dynamics," Dalton Trans. 2003, 1201-1217.

${ }^{14}$ A. C. Balazs and I. R. Epstein, "Emergent or just complex?," Science 325, 1632-1634 (2009).

${ }^{15}$ I. R. Epstein and K. Showalter, "Nonlinear chemical dynamics: Oscillations, patterns, and chaos," J. Phys. Chem. 100, 13132-13147 (1996). 
${ }^{16}$ I. R. Epstein, V. K. Vanag, A. C. Balazs, O. Kuksenok, P. Dayal, and A. Bhattacharya, "Chemical oscillators in structured media," Acc. Chem. Res. 45, 2160-2168 (2012).

${ }^{17}$ I. R. Epstein, "Predicting complex biology with simple chemistry," Proc. Natl. Acad. Sci. 103, 15727-15728 (2006).

${ }^{18}$ I. R. Epstein, "Coupled chemical oscillators and emergent system properties," Chem. Commun. 50, 10758-10767 (2014).

${ }^{19}$ A. S. Mikhailov and K. Showalter, "Control of waves, patterns and turbulence in chemical systems," Phys. Rep. 425, 79-194 (2006).

${ }^{20}$ S. K. Scott, Chemical Chaos (Clarendon Press, Oxford, 1991).

${ }^{21}$ I. R. Epstein, J. A. Pojman, and O. Steinbock, "Introduction: Selforganization in nonequilibrium chemical systems," Chaos 16, 037101 (2006).

${ }^{22}$ T. Yamaguchi, I. R. Epstein, M. Shimomura, and T. Kunitake, "Introduction: Engineering of self-organized nanostructures," Chaos 15, 047501 (2005).

${ }^{23}$ A. S. Mikhailov and K. Showalter, "Introduction to focus issue: Design and control of self-organization in distributed active systems," Chaos $\mathbf{1 8}$, 026101 (2008).

${ }^{24} \mathrm{~A}$. De Wit, K. Eckert, and S. Kalliadasis, "Introduction to the focus issue: Chemo-hydrodynamic patterns and instabilities," Chaos 22, 037101 (2012).

${ }^{25} \mathrm{G}$. Nicolis and I. Prigogine, Self-Organization in Nonequilibrium Systems (Wiley, New York, 1977).

${ }^{26}$ W. L. Ditto and K. Showalter, "Introduction: Control and synchronization of chaos," Chaos 7, 509-511 (1997).

${ }^{27}$ A. Hubler, R. Georgii, M. Kuchler, W. Stelzl, and E. Luscher, "Resonant stimulation of nonlinear damper oscillators by Poincare maps," Helv. Phys. Acta 61, 897-900 (1988).

${ }^{28}$ E. Ott, C. Grebogi, and J. A. Yorke, "Controlling chaos," Phys. Rev. Lett. 64, 1196-1199 (1990).

${ }^{29}$ W. L. Ditto, S. N. Rauseo, and M. L. Spano, "Experimental control of chaos," Phys. Rev. Lett. 65, 3211-3214 (1990).

${ }^{30}$ R. Roy, T. W. Murphy, T. D. Maier, Z. Gillis, and E. R. Hunt, "Dynamical control of a chaotic laser: Experimental stabilization of a globally coupled system," Phys. Rev. Lett. 68, 1259-1262 (1992).

${ }^{31}$ J. Singer, Y. Z. Wang, and H. H. Bau, "Controlling a chaotic system," Phys. Rev. Lett. 66, 1123-1125 (1991).

${ }^{32}$ V. Petrov, V. Gáspár, J. Masere, and K. Showalter, "Controlling chaos in the Belousov-Zhabotinsky reaction," Nature 361, 240-243 (1993).

${ }^{33}$ E. R. Hunt, "Stabilizing high-period orbits in a chaotic system: The diode resonator," Phys. Rev. Lett. 67, 1953-1955 (1991).

${ }^{34}$ B. Krauskopf, H. M. Osinga, E. J. Doedel, M. E. Henderson, J. Guckenheimer, A. Vladimirsky, M. Dellnitz, and O. Junge, "A survey of method's for computing (un)stable manifold of vector fields," Int. J. Bifurcation Chaos 15, 763-791 (2005).

${ }^{35}$ T. L. Carroll, I. Triandaf, I. Schwartz, and L. Pecora, "Tracking unstable orbits in an experiment," Phys. Rev. A 46, 6189-6192 (1992).

${ }^{36}$ Z. Gills, C. Iwata, R. Roy, I. B. Schwartz, and I. Triandaf, "Tracking unstable steady states-Extending the stability regime of a multimode laser system," Phys. Rev. Lett. 69, 3169-3172 (1992).

${ }^{37}$ V. Petrov, M. J. Crowley, and K. Showalter, "Tracking unstable periodicorbits in the Belousov-Zhabotinsky reaction," Phys. Rev. Lett. 72, 2955-2958 (1994).

${ }^{38}$ V. Petrov, S. Metens, P. Borckmans, G. Dewel, and K. Showalter, "Tracking unstable Turing patterns through mixed-mode spatiotemporal chaos," Phys. Rev. Lett. 75, 2895-2898 (1995).

${ }^{39}$ E. Mihaliuk, T. Sakurai, F. Chirila, and K. Showalter, "Feedback stabilization of unstable propagating waves," Phys. Rev. E 65, 065602 (2002).

${ }^{40}$ E. Mihaliuk, T. Sakurai, F. Chirila, and K. Showalter, "Experimental and theoretical studies of feedback stabilization of propagating wave segments," Faraday Discuss. 120, 383-394 (2002).

${ }^{41}$ T. Sakurai, E. Mihaliuk, F. Chirila, and K. Showalter, "Design and control of wave propagation patterns in excitable media," Science 296, 2009-2012 (2002).

${ }^{42}$ A. J. Steele, M. Tinsley, and K. Showalter, "Collective behavior of stabilized reaction-diffusion waves," Chaos 18, 026108 (2008).

${ }^{43}$ M. A. Dahlem, R. Graf, A. J. Strong, J. P. Dreier, Y. A. Dahlem, M. Sieber, W. Hanke, K. Podoll, and E. Schöll, "Two-dimensional wave patterns of spreading depolarization: Retracting, re-entrant, and stationary waves," Physica D 239, 889-903 (2010).

${ }^{44}$ M. A. Dahlem and E. P. Chronicle, "A computational perspective on migraine aura," Prog. Neurobiol. 74, 351-361 (2004).

${ }^{45}$ A. M. Zhabotinsky and A. N. Zaikin, in Oscillatory Processes in Biological and Chemical Systems II, edited by E. E. Sel'kov (Nauka, Puschino, 1971), p. 279.
${ }^{46}$ A. T. Winfree, Forsk. Framsteg 6, 9 (1971).

${ }^{47}$ A. Winfree, "Spiral waves of chemical activity," Science 175, 634-635 (1972).

${ }^{48}$ A. T. Winfree, "Scroll-shaped waves of chemical activity in three dimensions," Science 181, 937-939 (1973).

${ }^{49}$ W. Jahnke, W. E. Skaggs, and A. T. Winfree, "Chemical vortex dynamics in the Belousov-Zhabotinsky reaction and in the two-variable Oregonator model," J. Phys. Chem. 93, 740-749 (1989).

${ }^{50}$ R. J. Field and R. Noyes, "Oscillations in chemical systems. IV. Limit cycle behavior in a model of a real chemical reaction," J. Chem. Phys. 60, 1877-1884 (1974).

${ }^{51}$ G. S. Skinner and H. L. Swinney, "Periodic to quasiperiodic transition of chemical spiral rotation," Physica D 48, 1-16 (1991).

${ }^{52}$ D. Barkley, M. Kness, and L. S. Tuckerman, "Spiral wave dynamics in a simple model of excitable media-The transition from simple to compound rotation," Phys. Rev. A 42, 2489-2492 (1990).

${ }^{53}$ U. Storb, C. R. Neto, M. Bär, and S. C. Müller, "A tomographic study of desynchronization and complex dynamics of scroll waves in an excitable chemical reaction with a gradient," Phys. Chem. Chem. Phys. 5, 2344-2353 (2003)

${ }^{54}$ A. T. Winfree, S. Caudle, G. Chen, P. McGuire, and Z. Szilagyi, "Quantitative optical tomography of chemical waves and their organizing centers," Chaos 6, 617-626 (1996).

${ }^{55}$ T. Bansagi and O. Steinbock, "Negative filament tension of scroll rings in an excitable system," Phys. Rev. E 76, 045202 (2007).

${ }^{56}$ S. Alonso, F. Sagues, and A. S. Mikhailov, "Taming Winfree turbulence of scroll waves in excitable media," Science 299, 1722-1725 (2003).

${ }^{57}$ A. Azhand, J. F. Totz, and H. Engel, "Three-dimensional autonomous pacemaker in the photosensitive Belousov-Zhabotinsky medium," EPL 108, 10004 (2014).

${ }^{58}$ S. Dutta and O. Steinbock, "Topologically mismatched pinning of scroll waves," J. Phys. Chem. Lett. 2, 945-949 (2011).

${ }^{59}$ J. Maselko and K. Showalter, "Chemical waves on spherical surfaces," Nature 339, 609-611 (1989).

${ }^{60}$ J. Davidsen, L. Glass, and R. Kapral, "Topological constraints on spiral wave dynamics in spherical geometries with inhomogeneous excitability," Phys. Rev. E 70, 056203 (2004).

${ }^{61}$ O. Steinbock, P. Kettunen, and K. Showalter, "Anisotropy and spiral organizing centers in patterned excitable media," Science 269, 1857-1860 (1995)

${ }^{62}$ S. Jakubith, H. H. Rotermund, W. Engel, A. von Oertzen, and G. Ertl, "Spatiotemporal concentration patterns in a surface-reactionPropagating and standing waves, rotating spirals, and turbulence," Phys. Rev. Lett. 65, 3013-3016 (1990).

${ }^{63}$ S. Nettesheim, A. von Oertzen, H. H. Rotermund, and G. Ertl, "Reactiondiffusion patterns in the catalytic CO-oxidation on $\mathrm{Pt}(110)$-Front propagation and spiral waves," J. Chem. Phys. 98, 9977-9985 (1993).

${ }^{64}$ M. Kim, M. Bertram, M. Pollmann, A. von Oertzen, A. S. Mikhailov, H. H. Rotermund, and G. Ertl, "Controlling chemical turbulence by global delayed feedback: Pattern formation in catalytic $\mathrm{CO}$ oxidation on Pt(110)," Science 292, 1357-1360 (2001).

${ }^{65}$ J. Wolff, A. Papathanasiou, H. Rotermund, G. Ertl, M. Katsoulakis, X. $\mathrm{Li}$, and I. Kevrekidis, "Wave initiation through spatiotemporally controllable perturbations," Phys. Rev. Lett. 90, 148301 (2003).

${ }^{66}$ H. H. Rotermund, W. Engel, M. Kordesch, and G. Ertl, "Imaging of spatiotemporal pattern evolution during carbon-monoxide oxidation on platinum," Nature 343, 355-357 (1990).

${ }^{67}$ H. H. Rotermund, "Imaging of dynamic processes on surfaces by light," Surf. Sci. Rep. 29, 265-364 (1997).

${ }^{68} \mathrm{~K}$. Krischer, M. Eiswirth, and G. Ertl, "Oscillatory CO Oxidation on $\mathrm{Pt}(110)$-Modeling of temporal self-organization," J. Chem. Phys. 96, 9161-9172 (1992).

${ }^{69}$ M. Falcke, M. Bär, H. Engel, and M. Eiswirth, "Traveling waves in the CO oxidation on Pt(110)-Theory," J. Chem. Phys. 97, 4555-4563 (1992).

${ }^{70}$ M. Bär and M. Eiswirth, "Turbulence due to spiral breakup in a continuous excitable medium," Phys. Rev. E 48, R1635-R1637 (1993).

${ }^{71}$ P. De Kepper, A. Rossi, and A. Pacault, "Etude d'une réaction chimique périodique. Diagramme d'état de la réaction de Belousov-Zhabotinskii," C. R. Séances Acad. Sci., Ser. C 283, 371-375 (1976).

${ }^{72}$ P. De Kepper, I. R. Epstein, and K. Kustin, "A systematically designed homogeneous oscillating reaction-The arsenite-iodate-chlorite system," J. Am. Chem. Soc. 103, 2133-2134 (1981). 
${ }^{73}$ J. L. Hudson, M. Hart, and D. Marinko, “An experimental study of multiple peak periodic and nonperiodic oscillations in the BelousovZhabotinskii reaction," J. Chem. Phys. 71, 1601-1606 (1979).

${ }^{74}$ W. Y. Tam, W. Horsthemke, Z. Noszticzius, and H. L. Swinney, "Sustained spiral waves in a continuously fed unstirred chemical reactor," J. Chem. Phys. 88, 3395-3396 (1988).

${ }^{75}$ P. De Kepper, I. R. Epstein, K. Kustin, and M. Orbán, "Batch oscillations and spatial wave patterns in chlorite oscillating-systems," J. Phys. Chem. A 86, 170-171 (1982).

${ }^{76}$ V. Castets, E. Dulos, J. Boissonade, and P. De Kepper, "Experimental evidence of a sustained standing Turing-type nonequilibrium chemical pattern," Phys. Rev. Lett. 64, 2953-2956 (1990).

${ }^{77}$ W. Y. Tam, J. A. Vastano, H. L. Swinney, and W. Horsthemke, "Regular and chaotic chemical spatiotemporal patterns," Phys. Rev. Lett. 61, 2163-2166 (1988)

${ }^{78} \mathrm{P}$. Blanchedeau, J. Boissonade, and P. De Kepper, "Theoretical and experimental studies of spatial bistability in the chlorine-dioxide-iodide reaction," Physica D 147, 283-299 (2000).

${ }^{79}$ I. Lengyel and I. R. Epstein, "Modeling of Turing structures in the chlorite-iodide-malonic acid-starch reaction system," Science 251, 650-652 (1991).

${ }^{80}$ I. Lengyel and I. R. Epstein, "A chemical approach to designing Turing patterns in reaction-diffusion systems," Proc. Natl. Acad. Sci. 89, 3977-3979 (1992).

${ }^{81}$ J. Horvath, I. Szalai, and P. De Kepper, "An experimental design method leading to chemical Turing patterns," Science 324, 772-775 (2009).

${ }^{82} \mathrm{~V}$. K. Vanag and I. R. Epstein, "Pattern formation in a tunable medium: The Belousov-Zhabotinsky reaction in an aerosol OT microemulsion," Phys. Rev. Lett. 87, 228301 (2001).

${ }^{83}$ T. K. De and A. Maitra, "Solution behavior of aerosol OT in non-polar solvents," Adv. Colloid Interface Sci. 59, 95-193 (1995).

${ }^{84}$ T. Bánsági, Jr., V. K. Vanag, and I. R. Epstein, "Tomography of reactiondiffusion microemulsions reveals three-dimensional Turing patterns," Science 331, 1309-1312 (2011).

${ }^{85}$ T. Bansagi and O. Steinbock, "Three-dimensional spiral waves in an excitable reaction system: Initiation and dynamics of scroll rings and scroll ring pairs," Chaos 18, 026102 (2008).

${ }^{86}$ V. K. Vanag and I. R. Epstein, "Inwardly rotating spiral waves in a reaction-diffusion system," Science 294, 835-837 (2001).

${ }^{87}$ V. K. Vanag and I. R. Epstein, "Dash waves in a reaction-diffusion system," Phys. Rev. Lett. 90, 098301 (2003).

${ }^{88} \mathrm{~V}$. K. Vanag and I. R. Epstein, "Segmented spiral waves in a reactiondiffusion system," Proc. Natl. Acad. Sci. U. S. A. 100, 14635-14638 (2003).

${ }^{89}$ V. K. Vanag and I. R. Epstein, "Localized patterns in reaction-diffusion systems," Chaos 17, 037110 (2007).

${ }^{90}$ M. K. Runyon, B. L. Johnson-Kerner, and R. F. Ismagilov, "Minimal functional model of hemostasis in a biomimetic microfluidic system," Angew. Chem., Int. Ed. 43, 1531-1536 (2004).

${ }^{91}$ M. Toiya, V. K. Vanag, and I. R. Epstein, "Diffusively coupled chemical oscillators in a microfluidic assembly," Angew. Chem., Int. Ed. 47, 7753-7755 (2008).

${ }^{92}$ J. Delgado, N. Li, M. Leda, H. O. Gonzalez-Ochoa, S. Fraden, and I. R. Epstein, "Coupled oscillations in a 1D emulsion of BelousovZhabotinsky droplets," Soft Matter 7, 3155-3167 (2011).

${ }^{93}$ M. Toiya, H. O. González-Ochoa, V. K. Vanag, S. Fraden, and I. R. Epstein, "Synchronization of chemical micro-oscillators," J. Phys. Chem. Lett. 1, 1241-1246 (2010).

${ }^{94}$ N. Tompkins, N. Li, C. Girabawe, M. Heymann, G. B. Ermentrout, I. R. Epstein, and S. Fraden, "Testing Turing's theory of morphogenesis in chemical cells," Proc. Natl. Acad. Sci. 111, 4397-4402 (2014).

${ }^{95}$ Nonlinear Dynamics in Polymeric Systems, ACS Symposium Series Vol. 869, edited by J. A. Pojman and Q. Tran-Cong-Miyata (American Chemical Society, Washington, DC, 2003).

${ }^{96}$ N. M. Chechilo, R. J. Khvilivitskii, and N. S. Enikolopyan, "On the phenomenon of polymerization reaction spreading," Dokl. Akad. Nauk SSSR 204, 1180-1181 (1972).

${ }^{97}$ J. A. Pojman and T. W. McCardle, "Functionally gradient polymeric materials," U.S. patent 6,313,237 (November 6, 2001).

${ }^{98} \mathrm{~S}$. Gregory, "Ultraviolet curable resin compositions having enhanced shadow cure properties," U.S. patent 6,245,827 (June 12, 2001).

${ }^{99}$ R. Yoshida, T. Takahashi, T. Yamaguchi, and H. Ichijo, "Self-oscillating gel,” J. Am. Chem. Soc. 118, 5134-5135 (1996)
${ }^{100}$ O. Tabata, H. Hirasawa, S. Aoki, R. Yoshida, and E. Kokufuta, "Ciliary motion actuator using self-oscillating gel," Sens. Actuators, A 95, 234-238 (2002).

${ }^{101}$ R. Yoshida, "Design of functional polymer gels and their application to biomimetic materials," Curr. Org. Chem. 9, 1617-1641 (2005).

${ }^{102}$ V. V. Yashin and A. C. Balazs, "Pattern formation and shape changes in self-oscillating polymer gels," Science 314, 798-801 (2006).

${ }^{103}$ P. Dayal, O. Kuksenok, and A. C. Balazs, "Using light to guide the selfsustained motion of active gels," Langmuir 25, 4298-4301 (2009).

${ }^{104}$ X. Lu, L. Ren, Q. Gao, Y. Zhao, S. Wang, J. Yang, and I. R. Epstein, "Photophobic and phototropic movement of a self-oscillating gel," Chem. Commun. 49, 7690-7692 (2013).

${ }^{105}$ Y. Zhang, N. Zhou, S. Akella, Y. Kuang, D. Kim, A. Schwartz, M. Bezpalko, B. M. Foxman, S. Fraden, I. R. Epstein, and B. Xu, "Active cross-linkers that lead to active gels," Angew. Chem., Int. Ed. 52, 11494-11498 (2013).

${ }^{106}$ J. Aldridge and E. K. Pye, "Cell density dependence of oscillatory metabolism," Nature 259, 670-671 (1976).

${ }^{107}$ S. De Monte, F. d'Ovidio, S. Dano, and P. G. Sorensen, "Dynamical quorum sensing: Population density encoded in cellular dynamics," Proc. Natl. Acad. Sci. 104, 18377-18381 (2007).

${ }^{108}$ M. B. Miller and B. L. Bassler, "Quorum sensing in bacteria," Annu. Rev. Microbiol. 55, 165-199 (2001).

${ }^{109}$ A. F. Taylor, M. R. Tinsley, F. Wang, Z. Huang, and K. Showalter, "Dynamical quorum sensing and synchronization in large populations of chemical oscillators," Science 323, 614-617 (2009).

${ }^{110}$ W. F. Paxton, A. Sen, and T. E. Mallouk, "Motility of catalytic nanoparticles through self-generated forces," Chem. Eur. J. 11, 6462-6470 (2005).

${ }^{111}$ W. F. Paxton, S. Sundararajan, T. E. Mallouk, and A. Sen, "Chemical locomotion," Angew. Chem., Int. Ed. 45, 5420-5429 (2006).

${ }^{112}$ Y. Wang, R. M. Hernandez, D. J. Bartlett, J. M. Bingham, T. R. Kline, A. Sen, and T. E. Mallouk, "Bipolar electrochemical mechanism for the propulsion of catalytic nanomotors in hydrogen peroxide solutions," Langmuir 22, 10451-10456 (2006).

${ }^{113}$ T. E. Mallouk and A. Sen, "Powering nanorobots," Sci. Am. 300, 72-77 (2009).

${ }^{114} \mathrm{~J}$. Howse, R. Jones, A. Ryan, T. Gough, R. Vafabakhsh, and R. Golestanian, "Self-motile colloidal particles: From directed propulsion to random walk," Phys. Rev. Lett. 99, 048102 (2007).

${ }^{115}$ H. Ke, S. R. Ye, R. L. Carroll, and K. Showalter, "Motion analysis of self-propelled Pt-silica particles in hydrogen peroxide solutions," J. Phys. Chem. A 114, 5462-5467 (2010).

${ }^{116}$ G. Rückner and R. Kapral, "Chemically powered nanodimers," Phys. Rev. Lett. 98, 150603 (2007).

${ }^{117}$ S. Ebbens, R. Jones, A. Ryan, R. Golestanian, and J. Howse, "Selfassembled autonomous runners and tumblers," Phys. Rev. E 82, 015304 (2010).

${ }^{118} \mathrm{~S}$. Thakur and R. Kapral, "Collective dynamics of self-propelled spheredimer motors," Phys. Rev. E 85, 026121 (2012).

${ }^{119}$ J. Palacci, S. Sacanna, A. P. Steinberg, D. J. Pine, and P. M. Chaikin, "Living crystals of light-activated colloidal surfers," Science 339, 936-940 (2013).

${ }^{120}$ V. Horvath, P. L. Gentili, V. K. Vanag, and I. R. Epstein, "Pulse-coupled chemical oscillators with time delay," Angew. Chem., Int. Ed. 51, 6878-6881 (2012).

${ }^{121}$ Y. Kuramoto and D. Battogtokh, "Coexistence of coherence and incoherence in nonlocally coupled phase oscillators," Nonlinear Phenom. Complex Syst. 5, 380 (2002).

${ }^{122}$ D. Abrams and S. Strogatz, "Chimera states for coupled oscillators," Phys. Rev. Lett. 93, 174102 (2004).

${ }^{123}$ S. Shima and Y. Kuramoto, "Rotating spiral waves with phaserandomized core in nonlocally coupled oscillators," Phys. Rev. E 69, 036213 (2004)

${ }^{124}$ D. M. Abrams and S. H. Strogatz, "Chimera states in a ring of nonlocally coupled oscillators," Int. J. Bifurcation Chaos 16, 21-37 (2006).

${ }^{125}$ D. Abrams, R. Mirollo, S. Strogatz, and D. Wiley, "Solvable model for chimera states of coupled oscillators," Phys. Rev. Lett. 101, 084103 (2008).

${ }^{126}$ E. A. Martens, C. R. Laing, and S. H. Strogatz, "Solvable model of spiral wave chimeras," Phys. Rev. Lett. 104, 044101 (2010).

${ }^{127}$ G. Bordyugov, A. Pikovsky, and M. Rosenblum, "Self-emerging and turbulent chimeras in oscillator chains,” Phys. Rev. E 82, 035205 (2010). 
${ }^{128}$ G. C. Sethia, A. Sen, and F. M. Atay, "Clustered chimera states in delaycoupled oscillator systems," Phys. Rev. Lett. 100, 144102 (2008).

${ }^{129}$ E. A. Martens, "Chimeras in a network of three oscillator populations with varying network topology," Chaos 20, 043122 (2010).

${ }^{130} \mathrm{M}$. Wolfrum and O. Omel'chenko, "Chimera states are chaotic transients," Phys. Rev. E 84, 015201 (2011).

${ }^{131}$ A. M. Hagerstrom, T. E. Murphy, R. Roy, P. Hovel, I. Omelchenko, and E. Schöll, "Experimental observation of chimeras in coupled-map lattices," Nat. Phys. 8, 658-661 (2012).

${ }^{132}$ M. R. Tinsley, S. Nkomo, and K. Showalter, "Chimera and phase-cluster states in populations of coupled chemical oscillators," Nat. Phys. 8, $662-665$ (2012).

${ }^{133}$ S. Kadar, T. Amemiya, and K. Showalter, "Reaction mechanism for light sensitivity of the Ru(bpy) ${ }_{3}{ }^{2+}$-catalyzed Belousov-Zhabotinsky reaction," J. Phys. Chem. A 101, 8200-8206 (1997).

${ }^{134}$ A. Taylor, P. Kapetanopoulos, B. Whitaker, R. Toth, L. Bull, and M. Tinsley, "Clusters and switchers in globally coupled photochemical oscillators," Phys. Rev. Lett. 100, 214101 (2008).

${ }^{135} \mathrm{M}$. Wickramasinghe and I. Z. Kiss, "Spatially organized dynamical states in chemical oscillator networks: Synchronization, dynamical differentiation, and chimera patterns," PLoS One 8, e80586 (2013).
${ }^{136} \mathrm{M}$. Wickramasinghe and I. Z. Kiss, "Spatially organized partial synchronization through the chimera mechanism in a network of electrochemical reactions," Phys. Chem. Chem. Phys. 16, 18360-18369 (2014).

${ }^{137}$ L. Schmidt, K. Schönleber, K. Krischer, and V. García-Morales, "Coexistence of synchrony and incoherence in oscillatory media under nonlinear global coupling," Chaos 24, 013102 (2014).

${ }^{138}$ K. Schönleber, C. Zensen, A. Heinrich, and K. Krischer, "Pattern formation during the oscillatory photoelectrodissolution of n-type silicon: Turbulence, clusters and chimeras," New J. Phys. 16, 063024 (2014).

${ }^{139}$ S. Nkomo, M. R. Tinsley, and K. Showalter, "Chimera states in populations of nonlocally coupled chemical oscillators," Phys. Rev. Lett. 110, 244102 (2013).

${ }^{140}$ E. A. Martens, S. Thutupalli, A. Fourriere, and O. Hallatschek, "Chimera states in mechanical oscillator networks," Proc. Natl. Acad. Sci. U. S. A. 110, 10563-10567 (2013).

${ }^{141}$ M. Bennett, M. F. Schatz, H. Rockwood, and K. Wiesenfeld, "Huygens's clocks," Proc. R. Soc. A 458, 563-579 (2002).

${ }^{142}$ G. von Kiedrowski, S. Otto, and P. Herewijn, "Welcome home, systems chemists," J. Syst. Chem. 1, 1-6 (2010).

${ }^{143}$ S. Ulam, Adventures of a Mathematician (Scribner, New York, 1976).

${ }^{144}$ COST Action Meeting-SYSCHEM 2014, San Sebastian-Donostia, Spain, 2014. 